
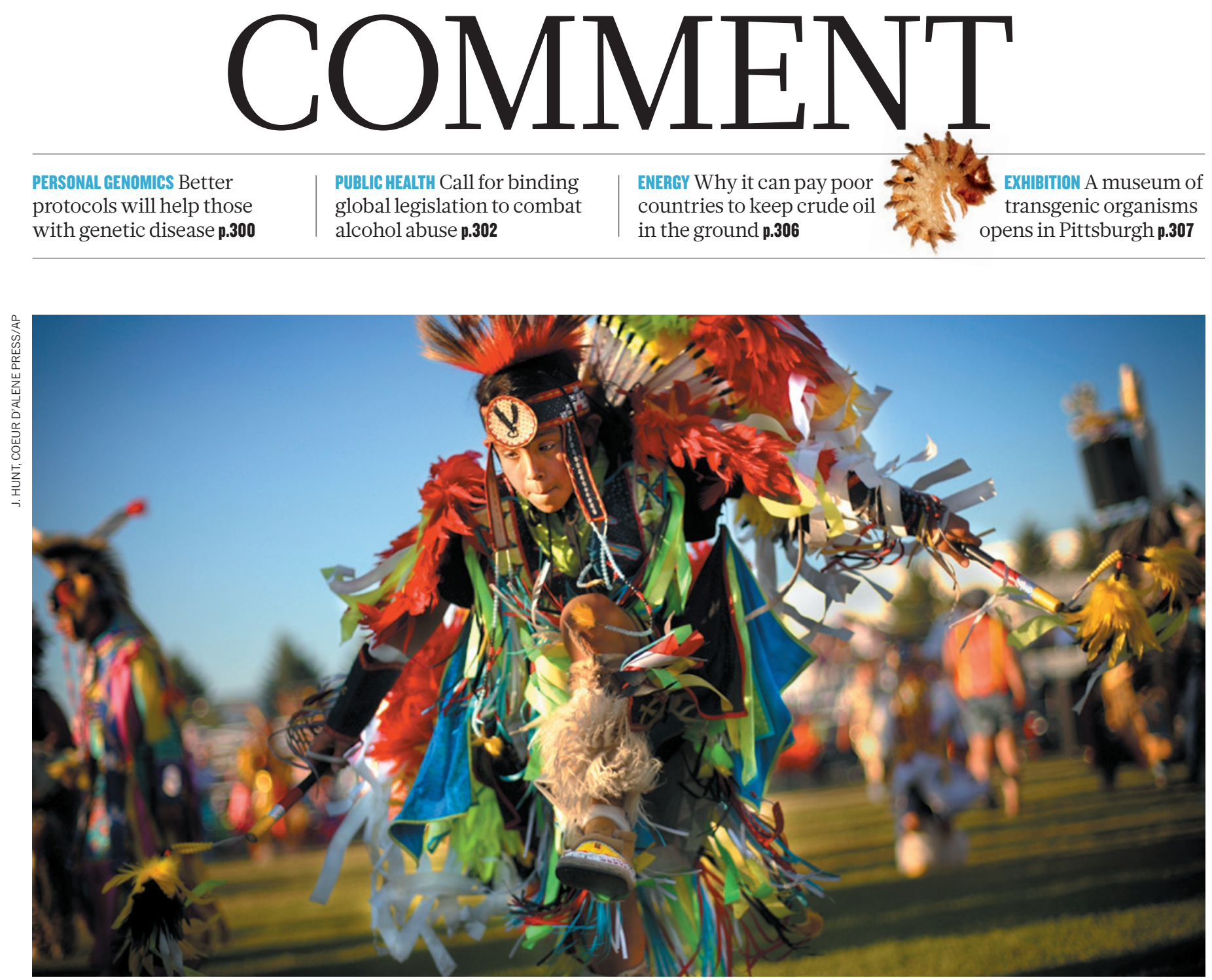

PERSONAL GENOMICS Better protocols will help those with genetic disease $\mathbf{p . 3 0 0}$ alcohol abuse $\mathbf{p . 3 0 2}$ countries to keep crude oil in the ground $\mathbf{p . 3 0 6}$ transgenic organisms pens in Pittsburgh p.307

In many indigenous cultures, including that of the Coeur d'Alene tribe, dance is used to transmit stories and teachings on to younger generations.

\title{
Adapted to culture
}

\section{Mark Pagel proposes that our ability to share and build on ideas is what made us human.}

W hat made us human? I propose that the development of a unique capacity for culture around 200,000 years ago was the defining event in the evolution of modern humans. A fastpaced evolutionary process emerged, which by 60,000 years ago had propelled modern humans out of Africa in small tribal societies to occupy and re-shape the world in just a few tens of thousands of years.

Culture became our strategy for survival. Our ability to learn from others and to transmit and build on knowledge, technology and skills might be the most potent trait the world has seen for converting new lands and resources into more humans. Whereas other species are confined to the environments their genes have adapted them to, we have adapted to nearly every environment on Earth.

\section{SUMMARY}

- A capacity for culture makes humans unique

- Transmitting technology and skills is our strategy for survival

- We became ultra-social through visual theft, the stealing of others' ideas - Language evolved from a need to negotiate

- Evolution has honed the range of our talents
Humans today, I suggest, are the descendants of those who were best at using this social juggernaut to advance their interests. The defining features of our nature - our ultra-sociality and language plus various innate talents and skills - arose as adaptations to living in the prosperous social environment of human culture, not from our shared history with other animals.

Our capacity for culture rests on two building blocks that together create an unbridgeable gap in evolutionary potential between us and all other species: social learning and 'theory of mind'. Through social learning, we can copy new behaviours merely by observing others. And $>$ 
with our theory of mind we can attribute mental states to others, allowing us to guess or understand their motives. We can then choose to copy the actions, ideas or inventions that have the best outcomes.

Both characteristics may be unique to humans. What looks like social learning in other animals could be little more than socially influenced learning that makes use of behaviours already present in an animal's repertoire ${ }^{1}$. For instance, chimpanzees manipulate things with their hands, so when one chimp uses a rock to crack open nuts, or a stick to fish for termites, another might be drawn to playing with rocks or sticks. That might by chance lead to a nut being cracked open or a termite retrieved. The reward would then reinforce the behaviour even though there was no direct imitation.

Some birds modify their behaviour when they know they are being observed by others of their species - as if 'aware' that the observer might use the knowledge it gains to its advantage. Thus, when a nutcracker bird sees another bird watching it while it hides its food, it will return alone later to hide the food in a new spot. This behaviour, also seen in other corvids, is intriguing, but it may just be a predisposition to respond to a learned behaviour; there is no good evidence beyond humans for a theory of mind ${ }^{2,3}$. In fact, most human two-year-olds show a greater understanding of others' beliefs than even adult apes do.

The upshot is that although some animals seem to have what we might call cultural 'traditions' - birds pecking at milk-bottle tops to get cream, for instance, or chimpanzees cracking open nuts with rocks - these habits do not evolve or improve over time ${ }^{3}$. Even after a million years, they will still be using the same techniques - unless they acquire true social learning and a theory of mind ${ }^{4}$.

By comparison, human societies evolve steadily through cumulative cultural adaptation. Our knowledge, skills and technologies accumulate improvements and produce variety, as people imitate each other, choose from and modify existing forms and combine objects to make new ones ${ }^{5}-$ when a shaped club was combined with a hand-axe, for example, the first hafted axe was born. The result is complex and varied culture that resembles animal cultural traditions about as much as a Bach cantata resembles a gorilla beating on its chest.

\section{VISUAL THEFT}

This capacity for improvement demanded changes that are not observed anywhere else in nature. Altruism is one example. Humans cooperate with unrelated individuals and perform acts of generosity that might not be repaid. We trade and exchange things, but we also hold doors open for people, give up seats on trains, contribute to charities and risk our lives by pulling someone from a burning building or fighting in a war. We are oddly group-focused: happy to wear silly matching shirts to sporting events, or paint our faces in the colours of our national flag, and keenly affected by the loss of our soldiers in battle. Who can forget images of Japan's fabled kamikaze warriors? You do not see this in chimpanzees.

In the rest of the animal kingdom, cooperation is generally confined to helping relatives. The the"Ourspecies's ory of kin selection history is the explains why: actions progressive that support your progressive triumph of cooperation over conflict." relatives benefit copies of your genes. But this theory is mute in the face of the human propensity to help strangers. We should therefore consider humans as 'ultra-social', having broken free of the usual genetic constraints on altruism.

Why do we behave in these ways? I suggest that around 160,000-200,000 years ago our capacity for culture created a social crisis to which ultra-sociality was the evolutionary solution. That crisis was visual theft ${ }^{4}$ - the capacity to steal others' ideas.

Because we can learn simply by watching others, knowledge is available to everyone and cultures can evolve and adapt at great speed. But if I watch which lure you use to catch a fish or how you haft a hand-axe, I benefit from your ingenuity as much as you do, possibly even more, because you had to spend time tinkering before you arrived at the solution I am now copying. I might even catch that fish before you do.

Thus, once a species acquires social learning, it becomes advantageous to keep the best ideas secret, lest they be stolen. This is illustrated today in our reluctance to share ideas - whether they be old family recipes, knowledge of fishing lures or new scientific or business plans - and also in our many patents and copyrights.

But hiding the best innovations would have brought cumulative cultural adaptation to a halt and caused our fledgling societies to collapse under the weight of suspicion and rancour. To avoid this outcome, we had to evolve the social rules and psychology that make it possible for people to exchange their ideas, knowledge and technology without undue fear of being exploited.

A great emphasis was then placed on demonstrating your own - and gauging others' - worthiness, because knowledge and technology were now held collectively by the social group, which wouldn't want to share them with cheats or competitors.

The many peculiar acts of altruism that describe our ultra-social nature evolved as costly ways for us to demonstrate our commitment, and thus our worthiness, to our cooperative group. The clearest way to show others that you are an altruist is to behave altruistically. The good reputations we earn attract altruism from others, which in turn grants us access to the material and social rewards of our communities.

We take our ultra-sociality for granted, but once such a system got going we had no choice but to become altruism 'show-offs', to compete with others for a slice of the cooperative pie. Our ultra-helpful nature is the altruism equivalent of a peacock's tail, except that the peacock uses his tail to attract a mate - we use our altruism to secure the spoils of cooperation.

Other unique features of our psychology, including our norms and morality, our expectation of fairness and our tendency towards 'moralistic aggression'

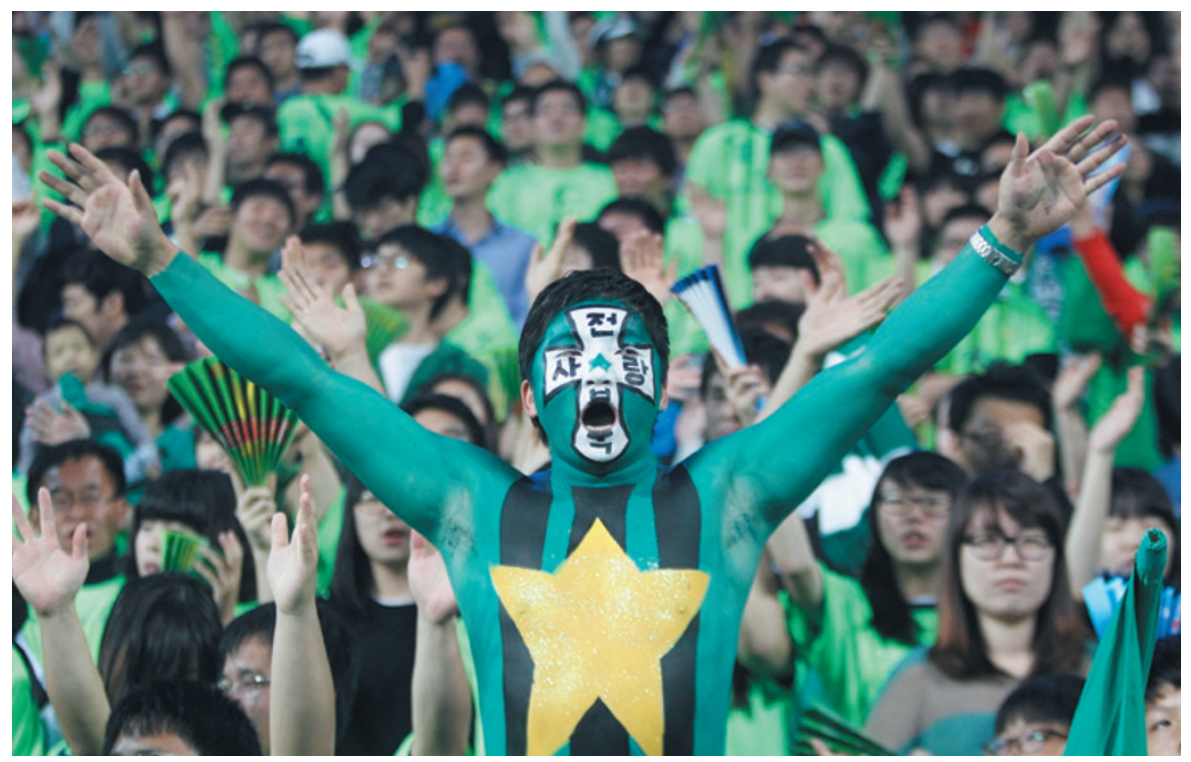

Humans are uniquely group-focused: many will deck themselves out in their team's colours. 
- punishing people who violate social rules - are emotions and social mechanisms we evolved to police those who might be tempted to exploit this fragile cooperative system.

\section{SOMETHING TO TALK ABOUT}

Human language differs from the grunts, chirrups, roars, odours, chest thumping and colourful displays of the rest of the animal kingdom in that it is compositional. We speak in sentences made from discrete sounds - words - that take the role of subjects, objects and verbs. Some animals make noun-like sounds - vervet monkeys can signal the approach of ground-based versus aerial predators - but only humans have been proven to use sentences.

Why? A number of ancient features of our anatomy and behaviour, such as our finely coordinated facial muscles or our primate tendencies to gesture, might have contributed to elements of our language ${ }^{6}$. But they do not explain why it evolved. I suggest that the complicated forms of cooperation and exchange we evolved to defuse the crisis of visual theft demanded a social technology for handling our deals, for coordinating our activities, for negotiating agreements and for broadcasting our reputations ${ }^{4}$. Language is that piece of social technology.

We acquired language because we were the only species with enough to talk about to pay for this expensive apparatus and the time and energy it takes to learn to use it. Lacking our social complexity, other animals don't need language, but human societies probably could not exist without it.

Even the simplest acts of exchange depend on language. Imagine you are good at making bows and I am good at making arrows, but our species has no language. I give you some arrows hoping you will give me bows in return. But you smile and, thinking my arrows are a gift, take them and walk off. I chase you, a scuffle ensues and I get stabbed with one of my own arrows. Now replay that scene with both actors having language: a cooperative and peaceful deal can be reached.

Research shows that the Neanderthals had the same version of a segment of DNA, known as FOXP2, that we do, and that has been implicated in the fine motor movements we use for speaking, leading many to suggest that Neanderthals, too, had language. Yet little in the archaeological record points to cumulative cultural adaptation in the Neanderthals ${ }^{7}$ - no musical instruments, no art, no fish-hooks or spear throwers. They did not even sew clothes. From the rule of visual theft, I suggest this dearth of culture tells us that the Neanderthals did not have language. Their human-like FOXP2 might have given them better communication abilities than other

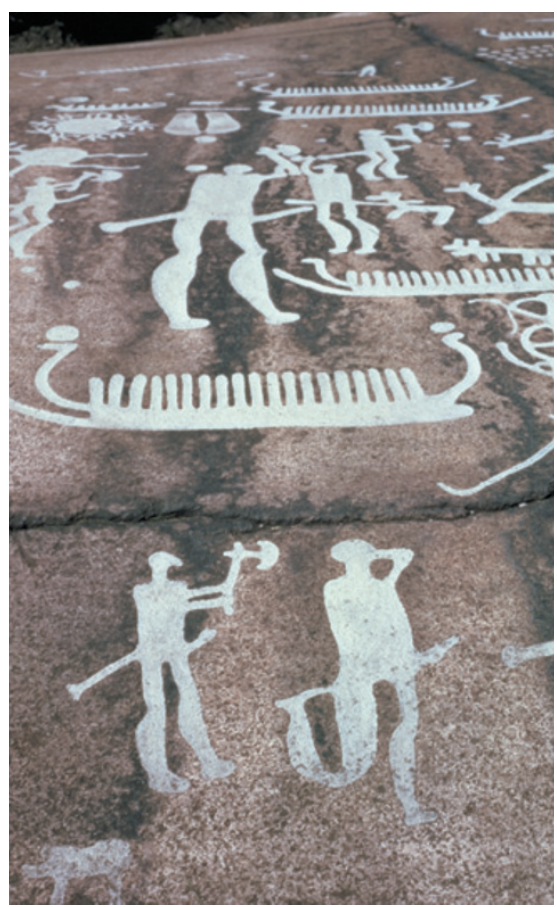

Our ability to build on and modify inventions has given us a selective advantage.

mammals, but in explaining the appearance of language we must look for the need for it, not just for pieces of anatomy or genes - some birds, for example, can mimic human speech, but do not share our version of FOXP2.

\section{DOMESTICATED BY CULTURE}

Humans have a surprisingly large range of abilities. Some of us are good at music, others at mathematics, design, language or sport, and all of these have been shown to have a significant genetic component ${ }^{8}$. Now, natural selection is the process by which some genetic varieties survive at the expense of others. It favours melodic singers among songbirds, and fast runners among lions and their antelope prey - poor singers remain lovelorn (and childless) and slow runners hungry or dead. We might therefore expect differences among us to get erased by natural selection. How, then, can we explain the diversity of human skills?

I believe that this variety is yet another consequence of our capacity for culture. Once our cooperative systems made it possible for people to exchange skills, goods and services, those who specialized at what they did best would have had the most to trade with others. In no other species is this possible, because no other species practises such a division of labour among unrelated individuals.

Our cultures domesticated and sorted us by our various talents, encouraging the skills to co-exist ${ }^{4}$. It is a scenario we should recognize, having inflicted it onto countless domesticated animals, notably dogs. Breeds ranging from chihuahuas to Newfoundlands bear the genetic marks of having evolved specialized temperaments, skills and morphologies in response to the social environment of human whims.

Our genes might have been equally content to specialize to the opportunities our societies created, and if so, this could have implications that are relevant for contemporary society. Most of us support the societal goal of ensuring equality of opportunity. But if people have different innate skills, then such a policy could produce a 'genetic meritocracy', a society differentiated by innate predispositions.

\section{THE MODERN WORLD}

There is evidence of an upturn beginning around 40,000 years ago in the degree of positive selection acting on our genes ${ }^{9}$, and involving hundreds of them ${ }^{10}$. It may not be an accident that this coincided with a flourishing of human culture as seen in an explosion of artefacts, art and musical instruments, and in our occupation of the world. These fast-evolving genes constitute our wiring for culture, and they can be identified using the same methods that isolate the genes that cause medical problems.

Modern societies differ vastly from the small tribes that once competed to occupy Earth. But the old psychology plays out well in our globalized multicultural world. Our species's history is the progressive triumph of cooperation over conflict as people recognized that cooperation could return greater rewards than endless cycles of betrayal and revenge. In a diverse world, the key to promoting this cooperation is to create among people a greater sense of trust and shared values that goes beyond the highly imprecise markers of ethnic or cultural differences. This is the social glue that has fostered our ultra-sociality and can continue to do SO. — SEE BOOKS \& ARTS P.304

Mark Pagel is professor of evolutionary biology in the School of Biological Sciences, University of Reading, UK. His latest book is Wired for Culture: Origins of the Human Social Mind (Norton/Penguin 2012). e-mail:m.pagel@reading.ac.uk

1. Laland, K. Curr. Biol. 18, R366-R370 (2008).

2. Heyes, C. Behav. Brain Sci. 21, 101-114 (1998).

3. Thornton, A. \& Clutton-Brock, T. Phil. Trans. R. Soc. B 366, 978-987 (2011).

4. Pagel, M. Wired for Culture (Norton/Penguin Press, 2012).

5. Ridley, M. The Rational Optimist: How Prosperity Evolves (Fourth Estate, 2010).

6. Fitch, W. T. The Evolution of Language (Cambridge University Press, 2010).

7. Stringer, C. The origin of our species (Allen Lane, 2011).

8. Pinker, S. The Blank Slate: The Modern Denial of Human Nature (Penguin Press, 2003).

9. Hawks, J. et al. Proc. Natl Acad. Sci. USA 104, 20753-58 (2007).

10.Williamson, S. H. et al. PLoS Genet. 3, e90 (2007) 